\title{
A new laser-ranged satellite for General Relativity and space geodesy: III. De Sitter effect and the LARES 2 space experiment
}

\author{
Ignazio Ciufolini ${ }^{1,2, a}$ (1), Richard Matzner ${ }^{3}$, Vahe Gurzadyan ${ }^{4}$, Roger Penrose ${ }^{5}$ \\ ${ }^{1}$ Dip. Ingegneria dell'Innovazione, Università del Salento, Lecce, Italy \\ 2 Centro Fermi, Rome, Italy \\ 3 Theory Group, University of Texas at Austin, Austin, USA \\ ${ }^{4}$ Center for Cosmology and Astrophysics, Alikhanian National Laboratory and Yerevan State University, Yerevan, Armenia \\ ${ }^{5}$ Mathematical Institute, University of Oxford, Oxford, UK
}

Received: 10 August 2017 / Accepted: 28 October 2017 / Published online: 1 December 2017

(C) The Author(s) 2017. This article is an open access publication

\begin{abstract}
In two previous papers we presented the LARES 2 space experiment aimed at a very accurate test of framedragging and at other tests of fundamental physics and measurements of space geodesy and geodynamics. We presented the error sources of the LARES 2 experiment, its error budget and Monte Carlo simulations and covariance analyses confirming an accuracy of a few parts in one thousand in the test of frame-dragging. Here we discuss the impact of the orbital perturbation known as the de Sitter effect, or geodetic precession, in the error budget of the LARES 2 frame-dragging experiment. We show that the uncertainty in the de Sitter effect has a negligible impact in the final error budget because of the very accurate results now available for the test of the de Sitter precession and because of its very nature. The total error budget in the LARES 2 test of frame-dragging remains at a level of the order of $0.2 \%$, as determined in the first two papers of this series.
\end{abstract}

\section{LARES 2 and an introduction to the de Sitter effect}

The LARES 2-LAGEOS space experiment is designed to achieve a new, accurate measurement of the General Relativistic frame-dragging due to the rotation of the Earth. Analytical estimates, covariance studies, and Monte Carlo simulations concur that the expected error level in this effect is of order $0.2 \%$, as shown in Refs. [1,2].

The two LAGEOS (Laser GEOdynamics Satellite) and the two LARES (Laser RElativity Satellite) are laser-ranged satellites. Satellite Laser Ranging (SLR) is the most accurate technique for measuring distances to the Moon [3] and to artificial satellites such as the LAGEOS and LARES satellites
[4-6]. Short-duration laser pulses are emitted, with different elevations, from lasers on the Earth towards a satellite and then reflected back to the emitting laser-ranging stations by the retro-reflectors on the satellite. The tracking data collected by the SLR network are analysed, organized and distributed by the International Laser Ranging Service (ILRS) [7]. By measuring the total round-trip travel time we are today able to determine the instantaneous distance of a retro-reflector on the LAGEOS and LARES satellites with a precision of a few millimetres [8]. Then, using orbital estimators, such as GEODYN, EPOSOC and UTOPIA, the orbit of the satellite is reconstructed and its six Keplerian orbital elements are determined with extremely high accuracy. For example the longitude of the ascending node can be determined with an uncertainty of a fraction of milliarcsecond that, over a long period of time, allows for extremely high accuracy in the measurement of the total nodal precession of a laser-ranged satellite. The LAGEOS satellites (LAGEOS and LAGEOS 2) [4] are spherical, made of heavy brass and aluminium, with a radius of $300 \mathrm{~mm}$ and about $406 \mathrm{~kg}$ in weight, completely passive and covered with retro-reflectors. LAGEOS and LAGEOS 2 have an essentially identical structure but they have different orbits. The semimajor axis of LAGEOS is $a=12270 \mathrm{~km}$, the eccentricity $e=0.004$ and the inclination $I=109.9^{\circ}$. The semimajor axis of LAGEOS 2 is $a_{I I}=12163 \mathrm{~km}$, the eccentricity $e_{I I}=0.014$ and the inclination $I_{I I}=52.65^{\circ}$. The LARES satellite [5], launched in 2012 by the Italian Space Agency (ASI) and ESA with the VEGA launch vehicle of ASI, ESA, AVIO and ELV, is spherical with a radius of $182 \mathrm{~mm}$ and a total mass of 386.8 $\mathrm{kg}$. It is a single spherical piece of a very dense tungsten alloy and it is covered with 92 retro-reflectors. The LARES orbital elements are semimajor axis $a_{L A R E S}=7820 \mathrm{~km}$,

\footnotetext{
a e-mail: ignazio.ciufolini@gmail.com
} 
orbital eccentricity $e_{L A R E S}=0.0008$, and orbital inclination $I_{L A R E S}=69.5^{\circ}$.

The LARES 2 satellite is planned for launch in 2019 with the new VEGA C launch vehicle of ASI, ESA, AVIO and ELV. It will be spherical with a radius of about $200 \mathrm{~mm}$ and a total mass of about $300 \mathrm{~kg}$. Its orbital elements will be semimajor axis $a_{L A R E S 2}=12270 \mathrm{~km}$, orbital inclination $I_{\text {LARES } 2}=70.16^{\circ}$ (supplementary to that of LAGEOS) and approximately null orbital eccentricity.

In addition to the frame-dragging, gravitomagnetic effect, whose test is the main objective of the LARES 2-LAGEOS space experiment, there is another general relativistic perturbation of an orbiting gyroscope, relative to an asymptotic inertial frame: the de Sitter or geodetic (or geodesic) precession [9] (see also [10]). This precession is due to the coupling between the velocity of a gyroscope orbiting a central body and the static part of the field (Schwarzschild metric) generated by the central mass.

The de Sitter precession can be derived by Fermi-Walker [11] transport along the worldline of a test-gyroscope. We first consider a spacelike spin four-vector $S^{\alpha}$ at each point of a timelike curve $x^{\alpha}(s)$ with tangent vector $u^{\alpha}$. We thus have $S^{\alpha} u_{\alpha}=0$. According to special relativistic kinematics and to the medium strong equivalence principle (all the laws of physics are the laws of special relativity in a local inertial frame [12-14,21]), the spin vector $S^{\alpha}$ obeys Fermi-Walker transport along the curve:

$S_{; \beta}^{\alpha} u^{\beta}=u^{\alpha}\left(a^{\beta} S_{\beta}\right) \equiv u^{\alpha}\left(u_{; \gamma}^{\beta} u^{\gamma} S_{\beta}\right)$,

where $a^{\beta} \equiv u^{\beta} ; \gamma u^{\gamma}$ is the four-acceleration of the testgyroscope and the semicolon denotes the covariant derivative. Suppose the timelike curve is a geodesic [12-15]. (Any test particle in the gravitational field of a massive body follows a timelike geodesic of the spacetime; a timelike geodesic path - world line - in spacetime's Lorentzian geometry is one that locally maximizes proper time, in analogy with the length-minimizing property of Euclidean straight lines. This is the case for a small body in free fall, affected only by gravitational forces.) Since a geodesic has zero four-acceleration: $u_{; \gamma}^{\beta} u^{\gamma}=0$, we then have $S_{; \beta}^{\alpha} u^{\beta}=0$. In this case the Fermi-Walker transport is just the parallel transport along the geodesic.

Therefore, in General Relativity, the orbital angular momentum of a test particle orbiting around a central body, assuming that both the test particle and the body follow geodesic motion, is parallel-propagated in the spacetime. Since geodesic motion is at the foundations of General Relativity, the generation of the best possible approximation to the free motion of a test particle, a spacetime geodesic, is a fundamental objective for experiments dedicated to the study of the spacetime geometry in the vicinities of a body and to high-precision tests of General Relativity and constraints on alternative gravitational theories.

A relevant problem is then the approximation to a geodesic that is provided by the motion of an extended body. In General Relativity [12-14], the problem of an extended body is profound, due not only to the non-linearity of the equations of motion, but also to the need to deal with the internal structure of the compact body, constructed of continuous media, where kinetic variables and thermodynamic potentials are involved. Furthermore, there may be intrinsic non-local effects arising from the internal structure of the extended body, such as tidal influences. Moreover, there are problems concerning the approximations that need to be made in order to describe a given extended body as a test particle moving along a geodesic. These problems are related to the fact that many of the common Newtonian gravitational concepts such as the centre of mass, total mass or size of an extended material body do not have well-defined counterparts in General Relativity [16].

The Ehlers-Geroch theorem [17] (generalizing the result in [18]) attributes a geodesic to the trajectory of an extended body with a small enough own gravitational field, if for a Lorentzian metric the Einstein tensor satisfies the so-called dominant energy condition [19], this tensor being non-zero in some neighbourhood of the geodesic and vanishing at its boundaries. This theorem affirms that small massive bodies move on near-geodesics and thus achieves a rigorous bridge from General Relativity to space experiments with small massive satellites, suggesting a high level of suppression of self-gravitational effects from the satellites own small gravitational field. This enables us to consider the motion of such small and massive satellite to be nearly geodesic and hence to provide a testing ground for general relativistic phenomena [20].

Indeed, given the extreme weakness of the gravitational interaction with respect to the other interactions of nature, the space environment is the ideal laboratory to test gravitational and fundamental physics. However, in order to test gravitational physics, a satellite must behave as nearly as possible as a test particle and must be as little as possible affected by non-gravitational perturbations, such as radiation pressure and atmospheric drag. In addition, its position must be determined with extreme accuracy. In space, a test particle can be realised in two ways: a small drag-free satellite or a small passive spacecraft with high density and an extremely small cross-sectional area to mass ratio. The best realisation of an orbiting test particle, with no drag-free system, is so far LARES [5].

For a passive satellite (with no drag-free system), the key characteristic that determines the level of attenuation of the non-gravitational perturbations is its density, reflected by the ratio between its cross-sectional area and its mass. Until the launch of LARES, the two LAGEOS satellites had the small- 
est ratio of cross-sectional area to mass of any other artificial satellite [5] and were the best available test particles around the Earth. The ratio of cross-sectional area to mass is approximately $0.0007 \mathrm{~m}^{2} / \mathrm{kg}$ for both satellites. LARES has a crosssectional area to mass ratio that is about 2.6 times smaller than that of LAGEOS. LARES 2 will have a cross-sectional area to mass ratio of approximately $0.0004 \mathrm{~m}^{2} / \mathrm{kg}$ larger only than that of LARES but will be orbiting at a much higher altitude with extremely small atmospheric drag perturbations. The extremely small cross-sectional area to mass ratio of the two LARES satellite, which is smaller than that of any other artificial satellite, and their special structure, a solid sphere with high thermal conductivity, ensure that the unmodelled nongravitational orbital perturbations are smaller than for any other satellite. This behaviour was experimentally confirmed using the first few months of laser-ranging observations [5].

Let us now study the general relativistic perturbations of a satellite such as LARES 2, the other non-gravitational perturbations, such as those due to radiation pressure and atmospheric drag, have been analysed in $[1,2]$. Furthermore, in order to study the de Sitter effect, we will only consider the spacetime metric generated in metric theories of gravitation by a static, non-rotating, mass. The accurate test of the general relativistic phenomena due to a stationary spacetime metric generated by a current of mass-energy, e.g., by a rotating mass, are the objective of the LARES 2 space experiment.

The spacetime metric generated by a matter distribution with rest-mass density $\rho$, but with a null mass-energy current density vector $\mathbf{j}$ (thus neglecting the dragging of inertial frames [21]), can be written, in a weak gravitational field, at the order of approximation beyond Newtonian theory (the post-Newtonian order) in terms of the standard Newtonian potential $U$, solution of $\Delta U=-4 \pi \rho$. In isotropic coordinates, we have $[14,22]$

$\mathrm{d} s^{2}=-\left(1-2 U+2 \beta U^{2}\right) \mathrm{d} t^{2}+(1+2 \gamma U) \delta_{i k} \mathrm{~d} x^{i} \mathrm{~d} x^{k}$

where $\beta$ and $\gamma$ are the two main post-Newtonian parameters, both equal to 1 in General Relativity, representing, respectively, the non-linearity in the superposition law for gravity of the gravitational interaction (interpretation valid in the socalled standard PPN gauge) and the amount of space curvature produced by a mass [14,22]. For simplicity, in Eq. (2), we have set equal to zero the other PPN parameters characteristic of preferred-frame, preferred-location and non-conservative theories. For $\beta$ and $\gamma$ equal to 1 , in standard non-isotropic coordinates, the metric is just the post-Newtonian approximation of the Schwarzschild metric $[13,14]$. To analyse the motion of a gyroscope, such as the orbital angular momentum of a test particle around the Earth, in the gravitational field of the Sun, we use the metric (2) where $U$ is the standard gravitational potential of the Sun. In the following treatment, we also include a non-gravitational acceleration a of the gyroscope.

To analyse the behaviour of the orientation of the spin of a test-gyroscope with respect to an asymptotic inertial frame [13], i.e. with respect to the distant stars (assuming that the universe is not rotating [14]), we introduce, at each point along the timelike world-line of the test-gyroscope, a local orthonormal frame [23]: $\lambda_{(\mu)}: g_{\alpha \beta} \lambda_{(\nu)}^{\alpha} \lambda_{(\mu)}^{\beta}=\eta_{\nu \mu}$. The index between parentheses, $\mu \epsilon(0,1,2,3)$, is the label of each vector of the local tetrad, or local vierbein (the indices between parentheses can be raised and lowered using $\eta^{\alpha \beta}$ and $\eta_{\alpha \beta}$, i.e. the Minkowski metric, and as usual the standard indices using $g^{\alpha \beta}$ and $g_{\alpha \beta}$.) By construction the timelike vector of this tetrad is the four-velocity of the test-gyroscope: $\lambda^{\alpha}{ }_{(0)} \equiv u^{\alpha}$ and, by construction, the spatial axes of the tetrad are non-rotating with respect to an asymptotic frame where $g_{\alpha \beta} \rightarrow \eta_{\alpha \beta}$. In other words the spatial axes are nonrotating with respect to a local orthonormal tetrad at rest in the asymptotically flat coordinate system of the metric (2). Therefore, the spatial axes of the tetrad are not FermiWalker transported along the timelike world line of the testgyroscope, but they are obtained at each point with pure Lorentz boosts-with no spatial rotations-between a local frame at rest in the coordinate system of the asymptotically flat, post-Newtonian, metric (2) and an observer moving with the test-gyroscope along the curve $x^{\alpha}(s)$ with four-velocity $u^{\alpha}$. The spatial axes $\lambda_{(i)}$ of this local frame may be thought of as physically realized by three orthonormal telescopes, always pointing towards the same distant stars fixed with respect to the asymptotic inertial frame where $g_{\alpha \beta} \longrightarrow \eta_{\alpha \beta}$ (neglecting aberration, which produces periodic variations in the pointing direction, but which averages out over one orbit). The vector $S^{\alpha}$ may be thought of as physically realized by the spacelike angular momentum vector of a spinning particle or test-gyroscope. Since $S^{\alpha}$ is a spacelike vector, $S^{\alpha} u_{\alpha}=0$, in the local frame $\lambda_{(\alpha)}$ we get : $S^{(0)}=0$. After some calculations, we finally get, at the post-Newtonian order,

$$
\begin{aligned}
& \frac{\mathrm{d} S_{(i)}}{\mathrm{d} s}=\epsilon_{(i)(j)(k)} \dot{\Omega}_{(j)} S_{(k)} \\
& \dot{\Omega}_{(j)}=\epsilon_{(j)(l)(m)}\left(-\frac{1}{2} v_{l} a_{m}+\left(\frac{1}{2}+\gamma\right) v_{l} U_{, m}\right)
\end{aligned}
$$

where $\epsilon_{(i)(j)(k)}$ is the Levi-Civita pseudotensor and the comma ${ }_{, m}$ denotes the standard partial derivative with respect to $x^{m}$ and in standard vector notation

$$
\begin{aligned}
\frac{\mathrm{d} \mathbf{S}}{\mathrm{d} s} & \equiv \dot{\mathbf{\Omega}} \times \mathbf{S} \\
\dot{\boldsymbol{\Omega}} & =-\frac{1}{2} \mathbf{v} \times \mathbf{a}+\left(\frac{1}{2}+\gamma\right) \mathbf{v} \times \nabla \mathbf{U}
\end{aligned}
$$

where $\mathbf{S} \equiv\left(S_{\alpha} \lambda_{(i)}^{\alpha}\right)$ and $\nabla$ is the standard gradient operator. 
This is the precession of a gyroscope with respect to an asymptotic inertial frame in the spacetime metric generated by a static distribution of matter. The first term of (4) is the Thomas precession, due to the non-commutativity of nonaligned Lorentz transformations and to the non-gravitational acceleration a. The second term is the de Sitter or geodetic precession [9] (see also [10]), which may be interpreted $[13,14,22]$ as due to a part (contributing with $\frac{1}{2}$ ) analogous to the Thomas precession, arising from the non-commutativity of non-aligned Lorentz transformations of special relativity and to the gravitational acceleration $\nabla U$, that is, due to Fermi-Walker transport and to the gravitational acceleration (which might be derived even in the flat spacetime of special relativity), plus a part (contributing with $\gamma$ ) due to Fermi-Walker transport and to the space curvature of General Relativity measured by the $\gamma$ parameter. In other words the de Sitter precession is the sum of two parts. One part, with factor $\frac{1}{2}$, is due to the time-time component of the metric tensor: $g_{00}$ (in standard post-Newtonian coordinates). If one writes $g_{00} \simeq-1+2 U-2 \beta U^{2}$, this effect is due to the second term in $g_{00}$. The other part, parametrized by $\gamma$ (equal to 1 in General Relativity), is due to the spatial curvature measured by $\gamma$ in the space-space components of the metric $g_{i j} \simeq(1+2 \gamma U) \delta_{i j}$. This effect was discovered in 1916 by de Sitter [9] (see also [10])

Thus, in the weak field, slow motion limit, the de Sitter precession of a gyroscope orbiting a central mass $M$, where $U=M / r$ is given by:

$$
\begin{aligned}
\dot{\boldsymbol{\Omega}}^{\text {de Sitter }} & =-\left(\frac{1}{2}+\gamma\right) \mathbf{v} \times \mathbf{r} \frac{M}{r^{3}} \\
& \cong(-19.2 \text { milliarcsec/year }) \hat{\mathbf{n}}
\end{aligned}
$$

where $\mathbf{v}$ is the velocity of the orbiting gyroscope, $\mathbf{r}$ the distance from the central mass to the gyroscope and $M$ the mass of the central body as measured in the weak field region. For an Earth-satellite gyroscope orbiting the Sun, this precession is about an axis perpendicular to the ecliptic plane defined by the unit vector $\hat{\mathbf{n}}$. The orbital plane of a satellite orbiting the Earth, such as LARES 2, LAGEOS or the Moon, may be thought of as a huge gyroscope orbiting the Sun and is thus affected by the solar de Sitter precession. Thus the solar de Sitter precession changes the nodal longitude of any satellite orbiting the Earth, measured relative to an asymptotic inertial frame, by:

19.2 milliarcsec/year $\times \cos 23.5^{\circ} \cong 17.6$ milliarsec/year

where $23.5^{\circ}$ is the obliquity of the ecliptic.

The de Sitter effect on a gyroscope due to the mass of the Sun has been accurately measured on the Moon-Earth "gyroscope" by Lunar Laser Ranging (see next section). The de Sitter effect on a gyroscope orbiting the Earth, due to the mass of the Earth, was measured by the Gravity Probe B experiment, but the Earth's de Sitter effect does not directly affect the LAGEOS-LARES 2 observation.

\section{Nature of the de Sitter effect and its impact in the accuracy of the LARES 2 experiment}

The de Sitter precession is a simple consequence of the gravitational field generated by a static, non-rotating, mass and has been measured, in weak field, by a huge number of highly accurate experiments (see, e.g. [14,22,24-26]). On the other hand, the frame-dragging effect is a consequence of the gravitational field of a rotating mass (such as a spinning black hole or the spinning Earth). For a discussion of the interpretation of the Lunar Laser Ranging observation of frame-dragging, see, e.g. [27]. The only tests of the spacetime solution of Einstein's General Relativity generated by a rotating mass (which has a key role to play in a number of astrophysical processes such as the emission of gravitational waves by a system of two coalescing spinning black holes) have been the LAGEOS plus LAGEOS 2, GP-B, and LARES tests, which have moderate accuracy. The LARES test is so far the most accurate test of frame-dragging with an uncertainty of about $5 \%$, and is aimed to reach an uncertainty of about $2 \%$. The LARES 2 experiment will improve the tests of framedragging to reduce the total error to a few parts in a thousand.

The de Sitter precession adds to the frame-dragging, but, as shown below, there exists no viable theory of gravitation that is both in agreement with all the existing tests of gravitational physics, and that predicts a solar system violation of the de Sitter precession at a level larger than $8.7 \times 10^{-6}$ (about 9 parts per million) of the value predicted by General Relativity, negligible at the level of the frame-dragging test accuracy.

The frame-dragging is independent of the de Sitter precession: there are indeed viable gravitational theories (such as the Chern-Simons theory and other $f(R)$ theories ([28]) which agree with all the gravitational tests except framedragging. The Chern-Simons gravitational theory is equivalent to heterotic string theories of type II with relevant implications for the explanation of one of the biggest riddles of physics today, the nature of dark-energy and quintessence, a mysterious form of energy calculated to constitute $70 \%$ of our universe. These theories predict a different outcome than General Relativity for frame-dragging around a rotating body, such as the Earth or a spinning black hole [28].

In the slow motion weak field situation appropriate for satellite motion near the Earth, both the Earth's dimensionless gravitational potential and the Sun's are small, of order $10^{-8}$ or less. Then the post Newtonian approximation holds for that satellite motion and the de Sitter precession, as 
derived in the previous section, can be written as the simple expression Eq. (5). As discussed in Sect. 1, the first term $\frac{1}{2}$ in Eq. (5) can be interpreted as the Thomas precession, an essentially Special Relativity effect under the central gravitational acceleration toward the mass $M$. The second term is proportional to the post Newtonian parameter $\gamma$ which describes the spatial curvature generated by the non-rotating central mass. Thus the de Sitter precession is a simple consequence of Special Relativity, of the extremely well tested equivalence principle (freely falling test particles follow timelike geodesics) and of the space curvature generated by a static mass, as parametrized by $\gamma$.

In the solar system, $\gamma$ has been measured by a large number of experiments. The most accurate determination of $\gamma$ so far is its measurement by the CASSINI spacecraft in the gravitational field of the Sun [29], with an accuracy of about $2.3 \times 10^{-5}$ (for a discussion of the CASSINI test of $\gamma$ see [30]); we emphasize that the orbital plane of the LARES 2 satellite will be affected by the de Sitter precession due to the mass of the Sun. From Eq. (5) this implies fractional error in the de Sitter precession of $\frac{2}{3}$ the fractional error in $\gamma$, yielding a fractional error about $1.53 \times 10^{-5}$ in the value of the de Sitter precession in the field of the Sun. The de Sitter precession perturbs the node of the LAGEOS satellite with a shift of 17.6 milliarcsec/year (see the previous section and [31]). Therefore the uncertainty in the de Sitter precession on the LAGEOS node is about $1.53 \times 10^{-5} \times 17.6$ milliarcsec/year $=2.7 \times 10^{-4}$ milliarcsec/year. The total frame-dragging effect on the node of LAGEOS is about 31 milliarcsec/year (as first calculated in [32,33]), giving a fractional error in the frame-dragging at a level of $\left(2.7 \times 10^{-4}\right.$ milliarcsec/year $) /(31$ milliarcsec/year) $=8.7 \times 10^{-6}$, which is negligible in the LARES 2-LAGEOS measurement of frame-dragging.

\section{Direct tests of the de sitter effect}

Instead of considering the tests of the space curvature parameter $\gamma$ one could consider only experiments directly measuring the de Sitter precession. The solar de Sitter precession was directly measured by the Lunar Laser Ranging (LLR) experiment, with an accuracy of about $5 \times 10^{-3}$ [26]. Translated to frame-dragging, this error by itself amounts to $\left(5 \times 10^{-3} \times 17.6\right.$ milliarcsec/year $) /(31$ milliarcsec/year $)$ $=0.28 \%$ error. Another test of the de Sitter precession was obtained by comparison of the Barycentric Celestial Reference System (BCRS) and Geocentric Celestial Reference System (GCRS) (see, e.g., [25], p. 699). Nevertheless, the group of the Leibniz Universität Hannover has recently published [34,35] a reanalyses of the LLR data yielding a value of the lunar geodetic precession measured to an accuracy of $1 \times 10^{-3}$. This gives a $0.06 \%$ contribution to the LARES 2 frame-dragging determination, still keeping the total (RSS) error budget at the level of about $0.2 \%$.

In conclusion, the results summarized in Table 1 show that the error due to the uncertainty in the de Sitter effect is negligible in the measurement of frame-dragging with LARES 2.

\section{Summary and conclusions}

To summarize the situation with respect to the error in knowledge of the de Sitter precession: (a) if we consider the solar system tests of General Relativity, mainly testing the gravitational field generated by the static, non-rotating, mass of the Sun, then the test of the space curvature parameter $\gamma$ (known with an uncertainty of about $2.3 \times 10^{-5}$ ) implies a fractional uncertainty of about $8.7 \times 10^{-6}$ in the test of framedragging by the LARES 2 experiment; (b) if one insists on considering only the direct tests of the de Sitter precession on Earth satellites by the Sun, then the recent results of reanalysis of the LLR data have an uncertainty of $1 \times 10^{-3}$ in the measurement of the de Sitter precession which implies a fractional uncertainty of about $0.06 \%$ in the test of framedragging by the LARES 2 experiment. In both cases, as summarized in Table 1, the error introduced by the uncertainty in de Sitter precession is negligible in the measurement of frame-dragging with LARES 2 and the total error budget in the LARES 2 test of frame-dragging remains at the level of about $0.2 \%$, as found in earlier papers in this series.

We gratefully acknowledge the Italian Space Agency for the support of the LARES and LARES 2 space missions under agreements No. 2017-23-H.0 and No. 2015-021R.0. We are also grateful to the International Ranging Ser-

Table 1 The error introduced by the de Sitter precession in the test of frame-dragging using LARES 2 and its impact in the total error budget

Error in the LARES 2 test of frame-dragging due Total error budget in the LARES 2 to the uncertainty in the de Sitter precession test of frame-dragging only

(a) Assuming the tests of space curvature due to the Sun static mass ( $\gamma$ accuracy by CASSINI: $2.3 \times 10^{-5}$ [29])

(b) Assuming only the tests of the de Sitter precession on the Moon with accuracy $1 \times 10^{-3}[34,35]$
$8.7 \times 10^{-6}$ error in the LARES 2 test of frame-dragging

$0.06 \%$ error in the LARES 2 test of frame-dragging $\approx 0.2 \%$ total error budget

$\approx 0.2 \%$ total error budget 
vice, ESA, AVIO and ELV. RM acknowledges NASA Grant NNX09AU86G and NSF Grant PHY-1620610.

Open Access This article is distributed under the terms of the Creative Commons Attribution 4.0 International License (http://creativecomm ons.org/licenses/by/4.0/), which permits unrestricted use, distribution, and reproduction in any medium, provided you give appropriate credit to the original author(s) and the source, provide a link to the Creative Commons license, and indicate if changes were made.

Funded by SCOAP ${ }^{3}$.

\section{References}

1. I. Ciufolini et al., A new laser-ranged satellite for General Relativity and Space Geodesy. I. Introduction to the LARES 2 space experiment. Eur. Phys. J. Plus 132, 336 (2017)

2. I. Ciufolini et al., A new laser-ranged satellite for General Relativity and Space Geodesy. II. Monte Carlo Simulations and covariance analyses of the LARES 2 Experiment. Eur. Phys. J. Plus 132, 337 (2017)

3. J.G. Williams, S.G. Turyshev, D.H. Boggs, Progress in lunar laser ranging tests of relativistic gravity. Phys. Rev. Lett. 93, 261101 (2004)

4. S.C. Cohen, P.J. Dunn (eds.), LAGEOS Scientific Results. J. Geophys. Res. 90(B11), 9215 (1985)

5. I. Ciufolini, A. Paolozzi, E.C. Pavlis, J. Ries, V. Gurzadyan, R. Koenig, R. Matzner, R. Penrose, G. Sindoni, Testing general relativity and gravitational physics using the LARES satellite. Eur. Phys. J. Plus 127, 127 (2012)

6. I. Ciufolini, A. Paolozzi, C. Paris, Overview of the LARES Mission: orbit, error analysis and technological aspects. J. Phys. Conf. Ser. 354(1), 012002 (2012)

7. M. Pearlman, J. Degnan, J. Bosworth, The international laser ranging service. Adv. Space Res. 30, 135-143 (2002)

8. J.C. Ries, R.J. Eanes, B.D. Tapley, G.E. Peterson, in Toward Millimeter Accuracy Proc. 13th Int. Laser Ranging Workshop, ed. by R. Noomen, S. Klosko, C. Noll, M. Pearlman (NASA CP 2003, 212248, NASA Goddard, Greenbelt, MD, 2003)

9. W. de Sitter, On Einstein's theory of gravitation and its astronomical consequences. Mon. Not. R. Astron. Soc. 77, 155-184, 481, 155184, 481 (1916)

10. A. Fokker, The geodesic precession; a consequence of Einstein's theory of gravitation. Proc. K. Ned. Akad. Wet. 23(5), 729-738 (1921)

11. E. Fermi, Sopra i Fenomeni che Avvengono in Vicinanza di una Linea Oraria. Atti Accad. Naz. Lincei Rend. Cl. Sci. Fis. Mat. Nat 31, 21-23, 51-52, 101-103 (1922)

12. S. Weinberg, Gravitation and Cosmology: Principles and Applications of the General Theory of Relativity (Wiley, New York, 1972)

13. C.W. Misner, K.S. Thorne, J.A. Wheeler, Gravitation (Freeman, San Francisco, 1973)

14. I. Ciufolini, J.A. Wheeler, Gravitation and Inertia (Princeton University Press, Princeton, 1995)

15. I. Ciufolini, Generalized geodesic deviation equation. Phys. Rev. D 34(4), 1014 (1986)
16. J. Ehlers, Survey of General Relativity Theory, in Relativity, Astrophysics and Cosmology, ed. by W. Israel (Reidel Publishing, 1973), pp. $1-125$

17. J. Ehlers, R. Geroch, Equation of motion of small bodies in relativity. Ann. Phys. 309, 232 (2004)

18. R. Geroch, P.S. Jang, Motion of a body in general relativity. J. Math. Phys. 16, 65 (1975)

19. S.W. Hawking, G.F.R. Ellis, The Large Scale Structure of SpaceTime (Cambridge University Press, Cambridge, 1975)

20. I. Ciufolini, V.G. Gurzadyan, R. Penrose, A. Paolozzi, Geodesic motion in General Relativity: LARES in Earths gravity, in: Low Dimensional Physics and Gauge Principles, Matinyan Festschrift (World Scientific, 2013), pp. 93-97

21. I. Ciufolini, Dragging of Inertial Frames. Nature 449, 41-47 (2007)

22. C.M. Will, Theory and Experiment in Gravitational Physics, 2nd edn. (Cambridge University Press, Cambridge, 1993)

23. J.L. Synge, Relativity the General Theory, 5th edn. (North Holland, Amsterdam, 1976)

24. S. Turyshev, Experimental tests of general relativity: recent progress and future directions. Phys. Uspekhi 52, 1-27 (2009)

25. S. Kopeikin, M. Efroimsky, G. Kaplan, Relativistic Celestial Mechanics of the Solar System (Wiley Online Library, New York, 2011)

26. J. Müller, L. Biskupek, F. Hofmann, E. Mai, Lunar Laser Ranging and Relativity, Frontiers in Relativistic Celestial Mechanicsvolume 2: Applications and Experiments, ed. S. Kopeikin (deGruyter, Berlin, Boston, 2014), pp. 103-156

27. S. Kopeikin, Y. Xie, Celestial reference frames and the gauge freedom in the post-Newtonian mechanics of the Earth Moon system. Celest. Mech. Dyn. Astron. 108, 245 (2010)

28. L. Smith, A.L. Erickcek, R.R. Caldwell, M. Kamionkowski, Effect of Chern-Simons gravity on bodies orbiting the Earth. Phys. Rev. D 77, 024015 (2008)

29. B. Bertotti, L. Iess, P. Tortora, A test of general relativity using radio links with the Cassini spacecraft. Nature 425, 374-376 (2003)

30. S. Kopeikin, A.G. Polnarev, G. Schäfer, I.Y. Vlasov, Gravimagnetic effect of the barycentric motion of the Sun and determination of the post-Newtonian parameter $\gamma$ in the Cassini experiment. Phys. Lett. A 367, 276-280 (2007)

31. I. Ciufolini, A Comprehensive Introduction to the Lageos Gravitomagnetic Experiment, from the Importance of the Gravitomagnetic Field in Physics to a Preliminary Error Budget. Int. J. Mod. Phys. A 4, 3083-3145 (1989)

32. I. Ciufolini, Theory and Experiments in General Relativity and other Metric Theories, Ph.D. Dissertation, University of Texas, Austin (Ann Arbor Publishers, Michigan, 1984)

33. I. Ciufolini, Measurement of the Lense-Thirring Effect on Lageos and another high altitude laser ranged artificial satellite. Phys. Rev. Lett. 56, 278-281 (1986)

34. F. Hofmann, Lunar Laser Ranging_-verbesserte Modellierung der Monddynamik und Schaetzung relativistischer Parameter, Ph.D. thesis (Leibniz Universität Hannover, 2017). http://www.dgk. badw.de.devweb.mwn.de/fileadmin/docs/c-797.pdf (Published in DGK series C, 797)

35. F. Hofmann, J. Müller, Relativistic tests with lunar laser ranging, to appear. Class. Quantum Gravity (2017). https://doi.org/10.1088/ $1361-6382 / \mathrm{aa} 8 \mathrm{f7} \mathrm{a}$ 